\title{
A Pre-registered Meta-analysis Based on Three Empirical Studies Reveals No Association Between Prenatal (Amniotic) Cortisol Exposure and Fluctuating Asymmetry in Human Infants
}

\author{
Will Bushell ${ }^{1} \cdot$ Martin Heil $^{2} \cdot$ Teresa Ventura $^{3} \cdot$ Manuel C. Gomes $^{4} \cdot$ Lisa M. Körner ${ }^{2} \cdot$ Judith Lawrenz $^{2} \cdot$ Nora K. Schaal $^{2}$. \\ Gareth Richards ${ }^{1}$ (D)
}

Received: 12 September 2020 / Accepted: 27 November 2020 / Published online: 15 January 2021

(c) The Author(s) 2021

\begin{abstract}
Developmental instability (DI) reflects an organism's inability to develop an ideal phenotype when challenged by genetic and environmental insults. DI can be estimated via the proxy measure of fluctuating asymmetry (FA), i.e., the small random deviations from perfect bilateral symmetry observed in the morphology of paired traits. The mechanisms involved in the genesis of FA in human populations are relatively unknown, though animal research indicates that hormonal processes may be involved. As maternal stress during pregnancy is detrimental to various developmental processes, elevated prenatal cortisol may represent a causal factor in the subsequent emergence of an asymmetrical phenotype. The main purpose of this pre-registered meta-analysis based on three empirical studies was to investigate whether mid-trimester amniotic cortisol levels predict subsequent FA in finger lengths of infants from Germany, Portugal, and the UK. No statistically significant relationships were observed, and meta-analytic combination of the effect size estimates yielded a null result. We did, however, detect significant positive correlations between the cortisol present in the amniotic fluid and maternal plasma in the Portuguese cohort, and observed that FA in the German cohort was significantly lower at 70-months than at either 9- or 20-months. Taken together, the current findings run contrary to animal research showing that elevated prenatal corticosterone exposure leads to increased FA. However, this may be because a single cortisol assay obtained via amniocentesis is an inadequate proxy for average gestational exposure, and/or that prenatal cortisol levels at an earlier (i.e., first rather than second trimester) stage of pregnancy is what explains variance in subsequent FA.
\end{abstract}

Keywords Amniocentesis $\cdot$ Cortisol $\cdot$ Developmental instability $\cdot$ Fluctuating asymmetry $\cdot$ Gestational stress $\cdot$ Maternal stress $\cdot$ Meta-analysis

Supplementary information The online version of this article (https://doi.org/10.1007/s11692-020-09523-9) contains supplementary material, which is available to authorized users.

Gareth Richards

gareth.richards@ncl.ac.uk

1 School of Psychology, Faculty of Medical Sciences, Newcastle University, 2.27 Ridley Building 1, Queen Victoria Road, Newcastle upon Tyne, UK

2 Institute of Experimental Psychology, Heinrich-Heine-University, Düsseldorf, Germany

3 Hospital Centre of Central Lisbon EPE, Rua Viriato, NOVA Medical SchoollFaculdade de Ciências Médicas, Campo Mártires da Pátria, Lisbon, Portugal

4 Faculty of Sciences, University of Lisbon, Campo Grande, Lisbon, Portugal

\section{Introduction}

Genetic and environmental stressors may cause a breakdown in developmental homeostasis which can result in departure from perfect bilateral symmetry in the morphology of paired traits. These random deviations, known as 'fluctuating asymmetry' (FA), are typically of small magnitude and exhibit a population mean value of 0 and a normal distribution around the mean (Palmer and Strobeck 1992; van Valen 1962). Whilst small, random deviations from perfect symmetry may not be intrinsically interesting in their own right, the use of FA as a phenotypic measure of developmental instability (DI) is common within a broad range of human and animal research (Møller and Swaddle 1997; Trivers et al. 1999; Van Dongen 2006; Van Dongen and Gangestad 2011). DI reflects an organism's inability to resist internal or environmental 
insult to biological development. In this sense, DI therefore refers to the degree of 'noise' present within genetic structures, whether of hereditary origin or as a property of environmental pressures. The utility of FA as a proxy measure of DI stems from the theory that both sides of bilateral traits develop under the control of identical genetic architecture. It can therefore be inferred that any deviations from perfect symmetry indicate disruption to ideal biological development and, therefore, reflect the organism's inability to resist such perturbations.

The genesis of a range of developmental conditions in humans may occur during prenatal development. It is therefore revealing that conditions for which an atypical developmental trajectory is implicated (e.g. autism [Boutrus et al. 2019; de Bruin et al. 2014]) have been linked with increased asymmetry. As ridge patterns on the fingertips are thought to form during weeks 14-22 of foetal development, and because the prenatal period is characterised by rapid brain growth (Stiles and Jernigan 2010), dermatoglyphic asymmetry is of particular interest to researchers in this field. For example, one recent study indicated that adolescents at increased risk of psychosis also exhibit a greater dermatoglyphic asymmetry than those at a lesser risk, though the effect was only small in size ( $r=0.23)$ (Russak et al. 2016). Despite such observations, few studies have investigated the developmental origins of FA in humans, and, although there is some suggestion that variations in hormonal exposure affect FA (Benderlioglu 2010), many gaps in our knowledge of the relevant processes remain.

Considering that prenatal stress can alter various developmental processes (Phelan et al. 2015; Ronald et al. 2011; van Os and Selten 1998), elevated foetal cortisol exposure may result in the subsequent emergence of an asymmetrical phenotype. Indeed, experimental animal research supports this premise (Eriksen et al. 2003; Newell-Morris et al. 1989; Satterlee et al. 2008). This research has also shown that elevated prenatal cortisol predicts a range of unfavourable developmental outcomes (for a review, see Weinstock 2005). It would clearly be unethical to experimentally manipulate prenatal hormone concentrations for research purposes in human populations, but preliminary data on pre-existing or naturally occurring stressors have been used to examine whether maternal stress during pregnancy is a predictor of FA in their offspring. For instance, Żądzińska et al. (2013) found that the sons of mothers who had experienced a traumatic event during pregnancy had elevated facial FA when measured at age 7-10 years. Similarly, King et al. (2009) reported that prenatal stress induced via a natural disaster (the 1998 Quebec Ice Storm) was associated with greater levels of asymmetry in fingertip ridge counts of infants whose mothers were exposed during gestational weeks 14-22. Additionally, those infants whose mothers had a higher subjective stress response to the ice storm showed significantly greater dermatoglyphic asymmetry than those whose mothers reported a less severe response. Whilst the mechanistic nature of the relationship between increased prenatal cortisol exposure and subsequent increases in FA of offspring is not clarified in these papers, the study of epigenetics may shed light on the phenomenon. For example, research indicates that maternal stress during pregnancy is associated with increased methylation of the NGFI-A binding site on the $\mathrm{NR}_{3} \mathrm{C}_{1}$ gene, which codes for glucocorticoid receptor (GC-R) sites (Oberlander et al. 2008; Palma-Gudiel et al. 2015). DNA methylation alters genetic expression largely by silencing promotor activity, and, as GC-R may act as a coactivator for STAT5-dependent transcription upon growth hormone stimulation (Tronche et al. 2004), it is therefore plausible that methylation of genetic structures such as these have downstream effects on bodily growth, possibly resulting in the emergence of an asymmetrical phenotype.

No published research has yet examined whether variations in foetal cortisol exposure are associated with FA in humans. Therefore, the current study examined whether mid-trimester cortisol levels measured via amniocentesis are associated with subsequent FA in finger lengths within three cohorts of children from the UK, Portugal, and Germany. Cortisol levels were measured in these cohorts at a time during which maternal stress is associated with increased methylation of crucial genetic structures (Oberlander et al. 2008) plausibly implicated in bodily growth (Tronche et al. 2004). It is through investigation of the theoretical effect of prenatal cortisol exposure on subsequent offspring FA (and by proxy, DI) that we can better understand the processes through which maternal stress impacts future generations. This is particularly relevant within an evolutionary framework, as maternal stress may cause epigenetic changes that modify the presentation of heritable phenotypes. Alongside plausible effects on FA as measured by bodily asymmetry, increased prenatal cortisol exposure is associated with a range of adverse developmental outcomes (Huizink et al. 2003) that could result in reduced fitness.

We pre-registered our analysis plan on the Open Science Framework (osf.io/am6zs) and made the following hypotheses: (1) within the German cohort, FA measured longitudinally would increase over time, (2) mid-trimester amniotic fluid cortisol levels would correlate positively with FA in each of the three cohorts, and (3) meta-analysis of the effect sizes estimated for each cohort would provide robust evidence of a positive correlation between amniotic cortisol exposure and FA. 


\section{Methods}

\section{Participants}

Children from three cohorts were included in the current research, all of whom were part of longitudinal studies of amniotic hormones. The German (Düsseldorf) cohort consists of children of mothers who underwent routine amniocentesis between 2010 and 2012 (Körner et al. 2019, 2020). Amniocentesis was prescribed for cases of increased risk of genetic and chromosomal abnormality due to advanced maternal age (mothers' age at birth: range $=22-48$ years, $M=38, S D=3.50)$. Of an initial sample of $n=244,39$ were removed for various reasons (amniotic cortisol not measured, no digit lengths recorded, digit lengths only recorded for one hand, left and right digits only measured at inconsistent time points [but never simultaneously]), which left a total sample size of $n=205$ healthy infants applicable for the current research goals (male $\mathrm{n}=105$; female $\mathrm{n}=100$ ).

For the UK (London) cohort (Glover et al. 2009; Richards et al. 2020; Sarkar et al. 2006, 2007), mothers were initially recruited sequentially from an amniocentesis clinic between December 2001 and January 2005. For the purposes of the current research, data from only 30 individuals were applicable (i.e., those for whom both amniotic cortisol and infant digit length measures were available). Here, gestational age at amniocentesis ranged between 15 and 25 weeks $(M=17.20, S D=2.37)$, maternal age at birth ranged between 25 and 43 years $(M=35.03$, $S D=4.18$ ) and maternal BMI ranged between 20.58 and $45.79(M=25.00, S D=4.95)$. Counts of stressful life events (SLE) during pregnancy were recorded in this cohort via a 26-item Stressful Life Events Questionnaire. Regarding ethnicity, mothers were recorded as $80 \%$ 'Caucasian', 3.33\% 'Asian-Indian sub-continent', 6.67\% 'Black', 6.67\% 'Middle Eastern' and 3.33\% 'Asian-Fareastern'. The women subsequently gave birth to 30 healthy infants (male $n=11$; female $n=19$ ), whose birthweights ranged between $2600 \mathrm{~g}$ and $3974 \mathrm{~g}(M=3403.14$, $S D=346.33$ ).

Mothers in the Portuguese (Lisbon) cohort were referred for karyotyping and recruited between December 2009 and June 2011 (Richards et al. 2019; Ventura et al. 2012, 2013). From these data, 99 applicable mothers were identified for the current research goals. Gestational age at amniocentesis ranged between 15 and 24 weeks $(M=17.14, S D=1.09)$, and maternal age at birth ranged between 26.8 and 43.5 years $(M=37.63, S D=2.76)$. In terms of ethnicity, $87 \%$ were recorded as 'Caucasian' and $13 \%$ simply as 'other'. Additionally (in contrast to both other cohorts), as part of the original research goals related to this sample, all mothers were assigned to one of three pre-procedure relaxation methods: (1) listening to music, (2) reading a magazine, or (3) simply sitting in the waiting room (control) (for further details, see Ventura et al. 2012). Measures of plasmatic cortisol were obtained from mothers twice, once half an hour prior to the procedure and once immediately before the procedure. 99 healthy infants (male $n=49$, female $n=50$ ) were born to these mothers, with birthweights ranging between $1725 \mathrm{~g}$ and $4610 \mathrm{~g}(M=3230.80, S D=515.87)$.

\section{Measurement of Amniotic Cortisol}

Across all cohorts, the level of amniotic cortisol was assayed from samples drawn at a single time point via amniocentesis. For the German cohort (Körner et al. 2019, 2020), amniotic fluid was analysed using liquid chromatography and tandem mass spectrometry (LCMS). This allows for accurate and specific simultaneous assessment of multiple biomarkers (Körner et al. 2019); further details on this hormonal analysis method are described by Rivet et al. (2018). For the UK cohort, an aliquot of up to $4 \mathrm{ml}$ of amniotic fluid surplus to clinical requirement was drawn for the original study and stored at $-80{ }^{\circ} \mathrm{C}$ until assay; total cortisol in amniotic fluid was then measured via radioimmunoassay (RIA), with cortisol having been extracted by dichloromethane and reconstituted prior to assay. For the Portuguese cohort, and similarly to that from Germany, an aliquot of 3-4 ml of amniotic fluid surplus to clinical requirement was drawn and samples were stored at $-80{ }^{\circ} \mathrm{C}$ until assay (Ventura et al. 2012); steroid hormone concentrations in the amniotic fluid samples were then measured externally at King's College London using LCMS.

\section{Measurement of Digit Lengths}

Finger lengths were measured in each of the three cohorts for the initial purpose of examining associations between amniotic testosterone and digit ratio (2D:4D), a putative indicator of prenatal sex hormone exposure (see Richards et al. 2020; Ventura et al. 2013). As measurements were available for both the right and left hands of participants from each cohort, the current study reused these data to determine FA. Finger lengths are commonly used for this purpose due to their representing clear examples of paired traits that can be measured with relative ease and accuracy. Additionally, although handedness effects could potentially result in asymmetric anatomical changes to the fingers (and indeed handedness shows a quadratic association with FA in adults; Yeo and Gangestad 1993), this is unlikely to present a serious confound in the current context due to the 
very young age of participants in each of the three cohorts studied.

Digit lengths (2D, 3D, 4D, and 5D) for both left and right hands were measured longitudinally in the Germany cohort at 5 distinct intervals: 5 months $(5 \mathrm{M}), 9$ months $(9 \mathrm{M})$, 20 months (20 M), 40 months (40 M), and 70 months $(70 \mathrm{M})$, and so we examined whether there were age-related changes in observed FA. Finger lengths were measured from photocopies of the ventral surface of the hand by two researchers using AutoMetric (DeBruine 2006), and were recorded in number of pixels. For the UK dataset, 2D and 4D measurements (given in $\mathrm{mm}$ ) of the left and right hands were taken at a single time-point, ranging between 15 and 19-months post-partum. Two researchers recorded finger lengths for this cohort using a combination of direct measures from participants' hands via Vernier calliper and indirect measures taken from photocopies. For the Portuguese cohort, 2D and 4D measurements (given in $\mathrm{mm}$ ) of the left and right hands were again available at a single interval, though much more immediately after birth than with the UK Cohort (ranging between 2.35 and 541.32-h post-partum). Due to the neonatal grasp reflex, the infants' hands were positioned to facilitate measurement of 2D and 4D (Ventura et al. 2013), and digit length measurements were recorded from photocopies by two independent researchers.

\section{Calculation of Composite Standardised Fluctuating Asymmetry}

For all cohorts, composite standardised fluctuating asymmetry (cFA) of 2D and 4D (and additionally 3D and 5D for the German cohort) was calculated as described by Voracek (2009, p. 107):

$\mathrm{FA}=\{\mathrm{Rd}|\mathrm{R} d-\mathrm{L} d| /[(\mathrm{R} d+\mathrm{L} d) / 2]\} / 2$

In this method, $d=2 \mathrm{D}$ and 4D (and 3D and 5D for the German dataset) lengths, respectively, and is expressed as a percentage of trait size (i.e., multiplied by 100). In other words, this calculation divides the absolute value of the difference in length between the right and left digits by the value of the summated right and left digit lengths, divided by 2 . This value is calculated separately for both $2 \mathrm{D}$ and 4D (and 3D and 5D in the case of the German cohort), and the final cFA value is taken as the mean of the 2D and 4D (and also 3D and 5D for the German cohort) values calculated (expressed as a percentage of trait size). This calculation should control for positive correlations between uncorrected unsigned FA and trait size that are sometimes reported. cFA was calculated from the second (2D) and fourth (4D) fingers for the UK and Portuguese cohorts. In the case of the German cohort, middle (3D) and little (5D) finger lengths were also available and so included in the cFA calculation.
To assess whether the heterogeneity in digit lengths used for cFA calculation may alter the results observed for this cohort, the analysis was run based on cFA calculated from only 2D and 4D length differentials as well. To that end, with respect to statistically significant results, no difference was observed in either univariate or multivariate analyses between the 2D and 4D only analyses and the 2D, 3D, 4D and 5D analyses. We therefore made the decision to proceed by using all available digits for the German cohort, as this is in-line with the pre-registered analysis plan and represents greater informational value.

\section{Statistical Analysis}

We used intra-class correlation coefficients to determine inter-rater reliability for finger length measurements (i.e., to verify that the measures taken were reliable/repeatable). To assess the extent to which measurement error had any attributable effect on observed variance in FA, we computed a linear mixed model with Participant ID as a random effect. From this, we were able to quantitatively measure how much of the variance in FA was due to commonalities between participants as the FA scores for each individual should be identical if there is no disagreement between researchers, and disagreement between researchers should logically only come in the form of measurement error. This was only possible for the UK and Portuguese cohorts, as only the averaged measures of two researchers were available for the German cohort. We then used paired samples $t$-tests to check for directional asymmetry. This is because if directional asymmetry is present, the variability of morphological asymmetry may be better explained by conditional factors (such as handedness) than by underlying DI. For this reason, although some posit mathematical approaches that take directional asymmetry into account (Graham et al. 1998), we use the parsimonious method of excluding from cFA those traits that exhibit directional asymmetry. We then used a Friedman test (followed by Wilcoxon signed ranks tests with Bonferroni correction) to examine associations between FA and age of measurement in the German cohort. Not only were we interested in how FA may change throughout development, but this procedure also helped inform decision making for subsequent analyses, such as whether it was appropriate to use a measure of average FA across all time-points or to use FA at the time-point with greatest sample size. As the initial research conducted with the Portuguese cohort included a pre-amniocentesis relaxation manipulation (Ventura et al. 2012), we used a Kruskal-Wallace test to determine whether this had any measurable effect on amniotic cortisol levels.

Preliminary testing revealed that some underlying assumptions of parametric analysis were violated even following logarithmic transformation, so associations between amniotic cortisol and FA were examined separately for each 
cohort via Kendall's $\tau$. Following this, bootstrapped (10,000 samples) multiple linear regression models were fitted with amniotic cortisol as the predictor, cFA as the outcome, and various demographic factors (dependant on data availability) as covariates. Maternal age was included as a covariate (all cohorts) because it has well documented associations with the viability and health of infants (Myrskylä and Fenelon 2012) and also because it may be relateded to offspring FA (Livshits et al. 1988). Sex of the infant was included (all cohorts) because FA during childhood is known to exhibit sexually differentiated developmental trajectories (Palestis and Trivers 2016). Birthweight (Portuguese and UK cohorts) and maternal BMI (UK cohort) were also considered as they are important predictors of future growth and developmental outcomes that could have downstream effects on FA (Jasienska 2013; Liu et al. 2019). The number of stressful life events (SLE) experienced during pregnancy was included as a covariate (UK cohort) because it provides an alternative index of maternal stress that is not dependant on cortisol concentrations assayed at a single time-point. Finally, pre-procedure relaxation method was included for the Portuguese cohort as, while no significant effect on amniotic cortisol concentration was observed, such effects could be difficult to detect due to their delayed and/or transient nature.

We used the R package metafor (Viechtbauer 2010) to conduct a random effects meta-analysis of the univariate effect size estimates observed for each cohort. Although not included in our pre-registration plan, we also performed an exploratory analysis (Kendall's $\tau$ ) to determine whether maternal plasmatic cortisol measured prior to amniocentesis

Table 1 ICCs and 95\% CIs for 2D and 4D lengths in the UK and Portuguese cohorts

\begin{tabular}{llllc}
\hline & L2D & L4D & R2D & R4D \\
\hline UK & & & & \\
ICC & 0.833 & 0.952 & 0.854 & 0.944 \\
95\% CI & $(0.67-0.92)$ & $(0.90-0.98)$ & $(0.71-0.93)$ & $(0.88-0.97)$ \\
Portugal & & & & \\
ICC & 0.964 & 0.961 & 0.972 & 0.960 \\
$95 \%$ CI & $(0.95-0.98)$ & $(0.94-0.97)$ & $(0.96-0.98)$ & $(0.94-0.97)$ \\
\hline
\end{tabular}

ICC intra-class correlation coefficient correlated with amniotic cortisol within the Portuguese cohort. All analyses were conducted using R and Rstudio v3.6.3, effects were considered statistically significant at $p<0.05$, and effect sizes were interpreted in accordance with Cohen (1988). The analysis plan, including non-parametric contingencies, was pre-registered on the Open Science Framework (osf.io/am6zs).

\section{Results}

\section{Inter-rater Reliability and Measurement Error}

Intra-class correlation coefficients (one-way random effects models with single rater unit) revealed excellent absolute agreement in digit measurements for the UK and Portuguese cohorts (Table 1) (analogous analyses were not conducted for the German cohort as we only had access to aggregated data). Consequently, digit length used to calculate cFA was taken as the arithmetic mean of the two measurements recorded by the separate researchers. Results from linear mixed effects models indicated that differences within individual measurements (and by assumption, measurement error) accounted for $20.86 \%$ and $14.98 \%$ of the variance in FA for the Portuguese and UK cohorts, respectively. Whilst these values are relatively high, they do still suggest that most variance is attributable to genuine differences between participants rather than measurement error (Table 2).

\section{Directional Asymmetry}

Paired samples $t$-tests revealed statistically significant differences in digit length measurements for $2 \mathrm{D}, 3 \mathrm{D}$ and $4 \mathrm{D}$ as measured at $40 \mathrm{M}$ between the right and left hands (all $p<0.05)$ in the German cohort, with digits for the right hand being longer on average than those of the left. No statistically significant differences in digit lengths between right and left hands were observed in the UK cohort for $2 \mathrm{D}, t(27)=0.063, p=0.95$, or $4 \mathrm{D}, t(27)=1.910$, $p=0.067$. However, a significant difference in $2 \mathrm{D}$ length was detected for the Portuguese cohort, $t(98)=4.845$, $p<0.001$, with digits on the right hand being larger than those of the left. Due to the observed pattern of results,
Table 2 Descriptive statistics for amniotic cortisol and FA across the three cohorts

\begin{tabular}{|c|c|c|c|c|c|c|}
\hline & \multicolumn{2}{|c|}{ Germany } & \multicolumn{2}{|l|}{ UK } & \multicolumn{2}{|c|}{ Portugal } \\
\hline & AvgFA & AmCort & $\mathrm{cFA}$ & AmCort & $\mathrm{cFA}$ & AmCort \\
\hline$N$ & 202 & 205 & 30 & 30 & 99 & 99 \\
\hline$M$ & 0.662 & 9.938 & 0.861 & 23.948 & 0.813 & 18.014 \\
\hline$S D$ & 0.307 & 4.517 & 0.551 & 8.400 & 0.616 & 8.326 \\
\hline
\end{tabular}

AmCort is measured in nmol/L; AvgFA average fluctuating asymmetry, AmCort amniotic cortisol concentration, $c F A$ composite standardised fluctuating asymmetry 
the decision was made to exclude all data pertaining to recordings made at the $40 \mathrm{M}$ interval when calculating cFA in the German cohort, and to base FA measures in the Portuguese cohort on 4D only (Palmer and Strobeck 1992, p. 65). However, we also assessed the impact of this decision via a sensitivity analysis including both $2 \mathrm{D}$ and $4 \mathrm{D}$ in cFA calculations. No difference was observed between the 4D only and 2D and 4D analyses in respect to statistical significance.

\section{Age-Related Changes in FA}

A Friedman test determined that cFA varied significantly across time-points, $\chi^{2}(3)=1215, p<0.001$, and post hoc pairwise comparisons (Wilcoxon signed-ranks tests with Bonferroni correction) revealed differences between $9 \mathrm{M}$

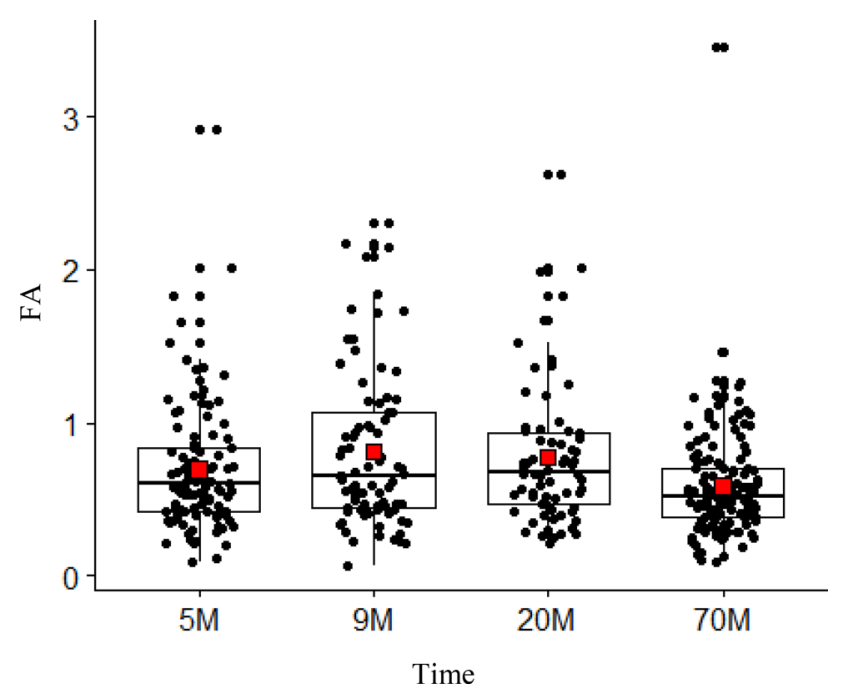

and $70 \mathrm{M}(Z=329, p=0.002)$, and between $20 \mathrm{M}$ and $70 \mathrm{M}(Z=1075, p=0.003)$. More specifically, FA was lower at $70 \mathrm{M}$ compared to $9 \mathrm{M}$ and $20 \mathrm{M}$ (Fig. 1).

\section{Relaxation Method and Amniotic Cortisol}

A Kruskal-Wallis test revealed no statistically significant effect of relaxation method on amniotic cortisol level within the Portuguese cohort, $\chi^{2}(2)=1.344, p=0.511$. The decision was therefore made to continue univariate analysis based on the full cohort (i.e., not split by relaxation method), but to include relaxation method as a covariate at the multivariate level.

\section{Amniotic Cortisol and FA}

\section{Univariate Analysis}

As there was no evidence of a linear relationship between time and FA in the German cohort, cFA scores for 2D, 3D, $4 \mathrm{D}$, and $5 \mathrm{D}$ at $5 \mathrm{M}, 9 \mathrm{M}, 20 \mathrm{M}$, and $70 \mathrm{M}$ were calculated for each individual. Not all participants were measured at all time points, so only applicable measurements were summated. Average FA (AvgFA) scores were therefore calculated by summing the FA scores for each time-point and then dividing by the number of time-points included in the calculation; AvgFA was then used as the final cFA score for the German cohort. For the other cohorts, cFA was calculated from 2D and 4D (UK) or just 4D measurements (Portuguese) as described earlier (see Table 3 for descriptive statistics). Kendall's $\tau$ yielded no statistically significant relationship across any of the three cohorts: Germany, $\tau_{\mathrm{b}}=0.048, p=0.311 ; \mathrm{UK}, \tau_{\mathrm{b}}=-0.067, p=0.620$; Portugal, $\tau_{\mathrm{b}}=-0.055, p=0.420$ (see Fig. 2).

Fig. 1 cFA measured longitudinally in the German cohort. Note. Horizonal bars indicate the median; red squares indicate the mean

Table 3 Covariates included in bootstrapped linear regression models for each cohort

\begin{tabular}{|l|l|l|l|}
\hline Variable & Germany & Portugal \\
\hline $\begin{array}{l}\text { Maternal age at } \\
\text { birth }\end{array}$ & & \\
\hline Sex of infant & & \\
\hline Birthweight & & \\
\hline $\begin{array}{l}\text { Pre-procedure } \\
\text { relaxation method }\end{array}$ & & \\
\hline Maternal BMI & & \\
\hline $\begin{array}{l}\text { Stressful life event } \\
\text { SLE) count during } \\
\text { pregnancy }\end{array}$ & & & \\
\hline
\end{tabular}




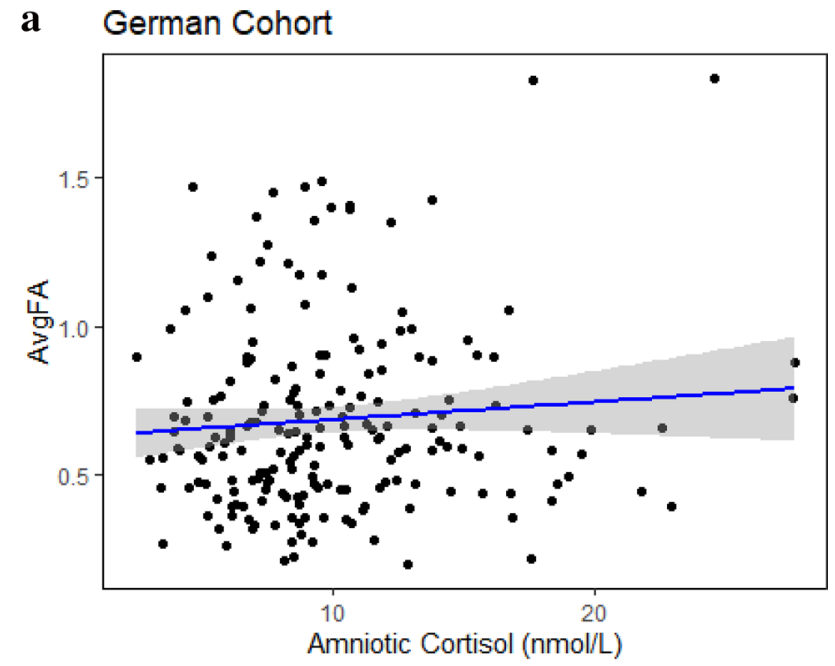

b UK Cohort

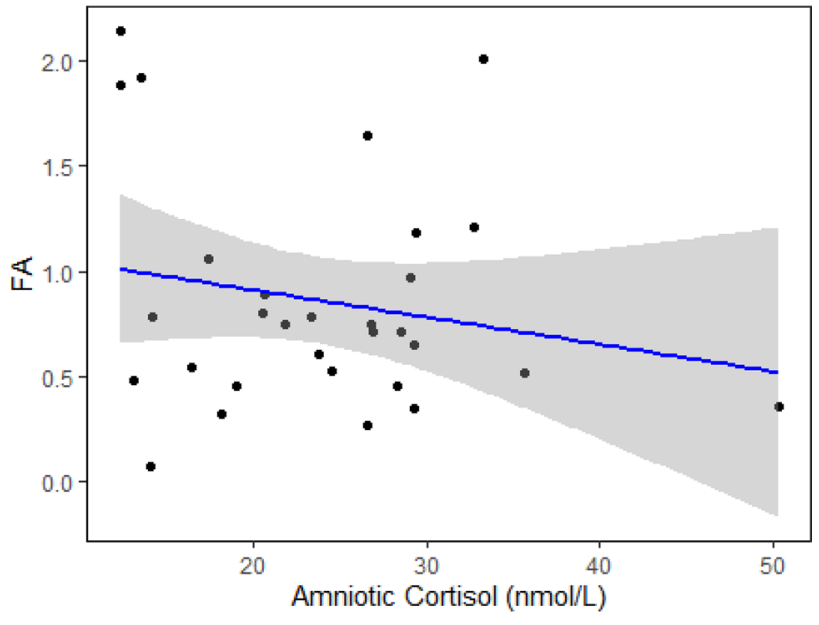

c Portuguese Cohort

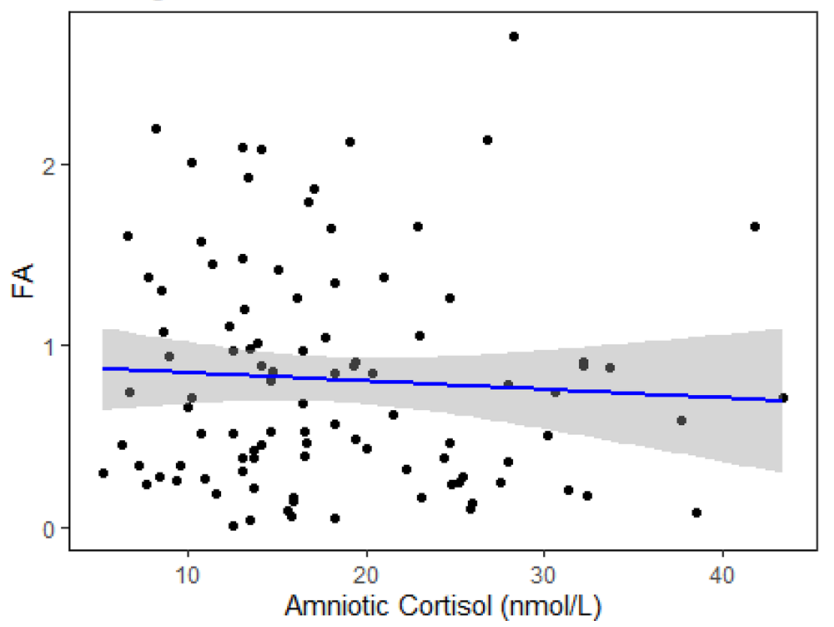

Fig. 2 Scatterplots of the association between amniotic cortisol and FA in the German (a $[r=0.075])$, UK (b $[r=-0.105])$, and Portuguese (c $[r=-0.086])$ cohorts

\section{Multivariate Analysis}

Bootstrapped multiple regression analyses (10,000 samples) were conducted to determine whether correlations between amniotic cortisol and FA would be revealed once important covariates (see Table 3 ) had been controlled for statistically. These analyses showed no statistically significant associations, as in all cases the bootstrapped $95 \%$ CIs overlapped 0 (see Table 4 for summary statistics).

\section{Meta-analysis of the relationship between amniotic cortisol and FA}

Random effects meta-analysis was performed to determine a weighted average effect size estimate for the level of association between amniotic cortisol and FA based on the correlation coefficients observed across the three cohorts. Effect sizes were converted from Kendall's $\tau$ to Pearson's $r$ using the following formula outlined by Walker (2003):

$r=\sin (.5 \pi \tau)$

As Pearson's $r$ is not normally distributed, these were then converted to Fisher's $z$ for meta-analysis, and the results back-calculated to $r$ for ease of interpretation (Borenstein et al. 2009). Heterogeneity was determined using a restricted maximum likelihood estimator, and no statistically significant difference in effect size was observed across the three studies, $\mathrm{Q}(2)=2.126, p=0.346$. The overall meta-analytic effect size estimate was not statistically significant, $r$ $(2)<0.001$ (95\% CI $-0.134,0.134), p=0.999$, suggesting no association between amniotic cortisol concentration and subsequent finger length FA (see Fig. 3 for forest plot).

\section{Association Between Cortisol in the Maternal Circulation and Amniotic Fluid}

Kendall's $\tau$ revealed that amniotic cortisol was positively correlated with maternal cortisol measured $30 \mathrm{~min}$ prior to $\left(\tau_{\mathrm{b}}=0.282, p<0.001\right)$ and immediately before amniocente$\operatorname{sis}\left(\tau_{\mathrm{b}}=0.275, p<0.001\right)$ (Fig. 4).

\section{Discussion}

The current study aimed to test the hypothesis that prenatal exposure to the stress hormone cortisol will correlate positively with fluctuating asymmetry (FA), a putative indicator of developmental instability (DI). To this end, mid-trimester cortisol measured from amniotic fluid samples collected for three cohorts of children in Germany, Portugal and the UK were correlated with standardised composite FA (cFA) calculated from finger length differentials for 2D and 4D (and 
Table 4 Summary statistics from bootstrapped (10,000 samples) multiple regression analyses for each cohort with amniotic cortisol as predictor and FA as outcome

\begin{tabular}{|c|c|c|c|c|c|c|c|c|}
\hline & Constant & AmCort & Maternal age & Sex of infant & Birthweight & $\begin{array}{l}\text { Pre-procedure } \\
\text { relaxation } \\
\text { method }\end{array}$ & $\begin{array}{l}\text { Maternal } \\
\text { BMI }\end{array}$ & SLE count \\
\hline \multicolumn{9}{|l|}{ Germany } \\
\hline Original data & 0.683 & 0.016 & -0.002 & 0.014 & & & & \\
\hline $\begin{array}{l}\text { Bootstrap } \\
\text { SE }\end{array}$ & 0.256 & 0.016 & 0.007 & 0.044 & & & & \\
\hline $\begin{array}{r}\text { Bootstrap } \\
95 \% \text { CI }\end{array}$ & $0.190,1.203$ & $-0.013,0.049$ & $-0.015,0.011$ & $-0.072,0.099$ & & & & \\
\hline \multicolumn{9}{|l|}{ Portugal } \\
\hline Original data & 0.089 & -0.006 & 0.016 & 0.007 & 0.0001 & 0.045 & & \\
\hline $\begin{array}{l}\text { Bootstrap } \\
\text { SE }\end{array}$ & 0.958 & 0.008 & 0.024 & 0.140 & 0.0001 & 0.108 & & \\
\hline $\begin{array}{r}\text { Bootstrap } \\
95 \% \text { CI }\end{array}$ & $-1.606,2.139$ & $-0.021,0.011$ & $-0.037,0.057$ & $-0.264,0.282$ & $\begin{array}{c}-0.0002 \\
0.0003\end{array}$ & $\begin{array}{c}-0.167 \\
0.256\end{array}$ & & \\
\hline \multicolumn{9}{|l|}{ UK } \\
\hline Original data & 0.203 & -0.014 & -0.024 & 0.452 & 0.0006 & & 0.033 & -0.163 \\
\hline $\begin{array}{l}\text { Bootstrap } \\
\text { SE }\end{array}$ & 1.904 & 0.018 & 0.030 & 0.232 & 0.0004 & & 0.027 & 0.082 \\
\hline $\begin{array}{r}\text { Bootstrap } \\
95 \% \text { CI }\end{array}$ & $-2.515,4.968$ & $-0.045,0.027$ & $-0.059,0.060$ & $-0.445,0.458$ & $\begin{array}{c}-0.0007 \\
0.0007\end{array}$ & & $\begin{array}{c}-0.053 \\
0.056\end{array}$ & $-0.160,0.164$ \\
\hline
\end{tabular}

Note Bootstrapped 95\% CIs are percentile intervals; AmCort amniotic cortisol concentration, SLE count number of stressful life events experienced during pregnancy

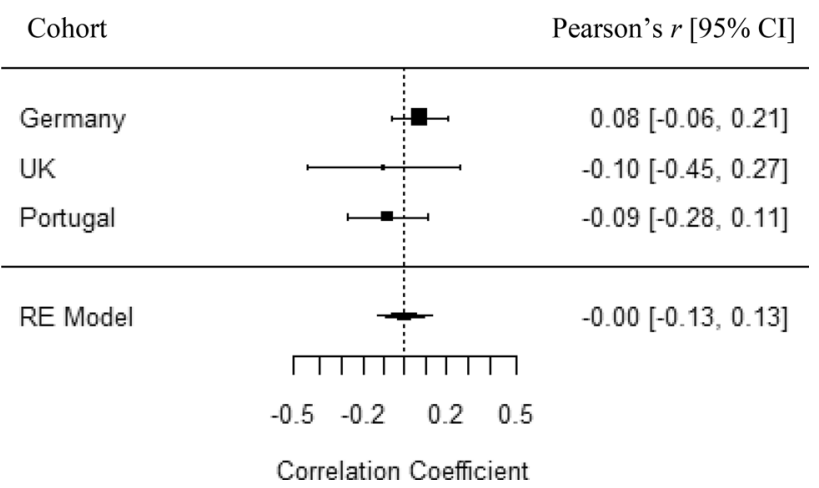

Fig. 3 Forest plot showing effect size (Pearson's $r$ ) estimates for association between amniotic cortisol exposure and $\mathrm{cFA}$

additionally 3D and 5D for the German cohort). Findings from the three cohorts were considered on an individual and meta-analytical basis, providing an in-depth and appropriately powered research framework. All hypotheses, analysis plans and contingencies related to this research were preregistered with OSF prior to the onset of statistical analysis, limiting our scope to infer statistical significance in nonpredetermined variables and plausibly reducing the probability of Type 1 errors. This is of particular importance when considering the effect of publication bias on a field in which the reporting of small magnitude effects is commonplace (Van Dongen and Gangestad 2011). Univariate, multivariate, and meta-analytic models revealed no significant association between these variables, providing no support for our hypothesis. Furthermore, only data from the German cohort provided results in the hypothesised direction $(n s)$. We also predicted that FA would increase as a function of age in the German cohort, though actually observed lower FA at 70 months than 9 and 20 months. The current findings suggest that variability in second trimester cortisol exposure observed in typically developing pregnancies is not an indicator of DI. This observation runs contrary to findings from the animal literature but may be explained by limitations associated with assaying cortisol from amniotic fluid and measuring FA solely from finger lengths.

\section{Age-Related Changes in FA}

Relatively little is known regarding how FA changes throughout development. Evidence from animal models indicates that variance in shape halves of skull morphology in two rodent species reduced over time (Zelditch et al. 2004). One explanation for this is that variation in phenotypic morphology is only rapidly accounted for later in growth as variation is continually generated but accumulates during early development as biological mechanisms that compensate for this variation are not yet functioning. A second explanation is that variation is only generated prenatally or during early development and is in fact rapidly compensated for. 

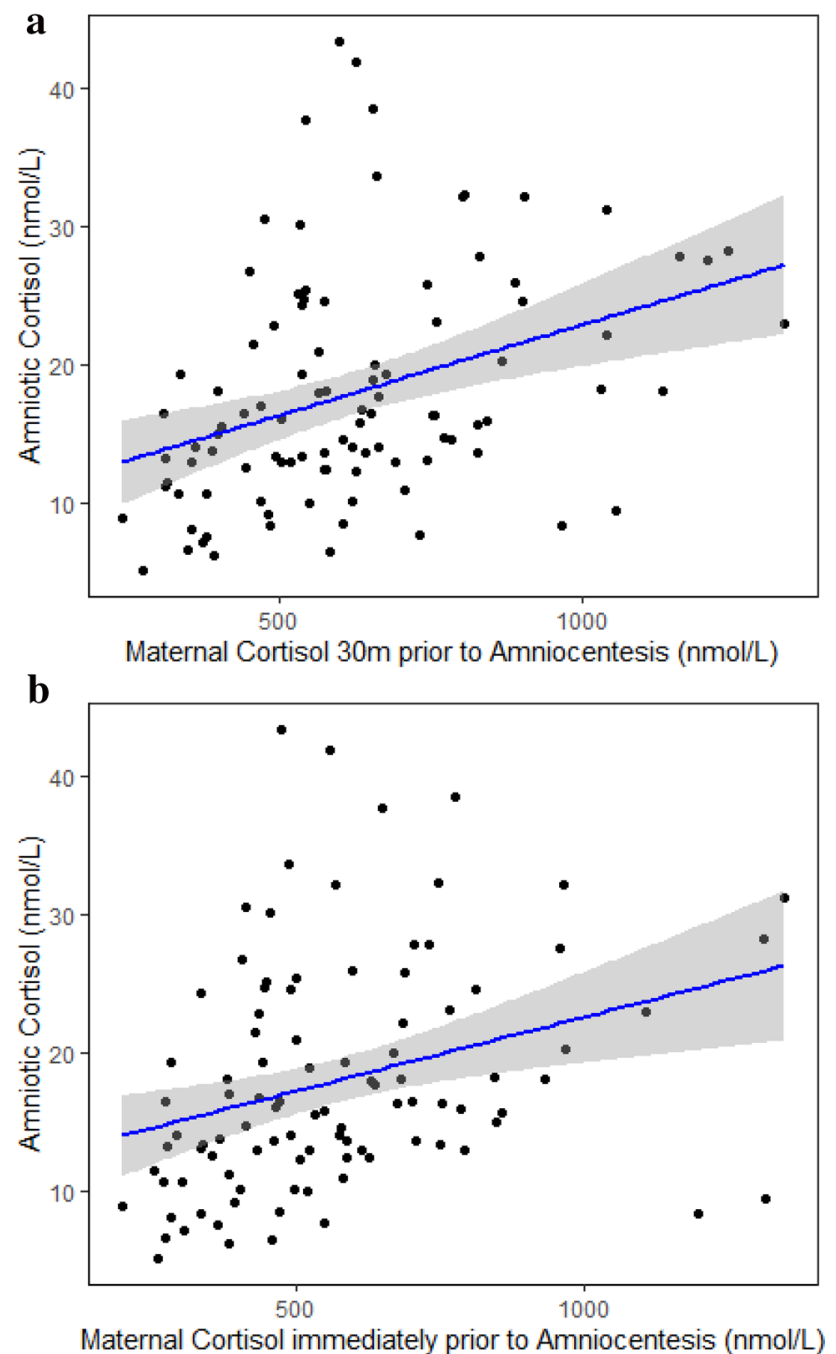

Fig. 4 Scatterplots showing correlation between amniotic cortisol and maternal plasmatic cortisol measured a $30 \mathrm{~min}$ before and $\mathbf{b}$ immediately before amniocentesis in the Portuguese cohort

However, as no new variation is introduced after this period, variability in phenotypic morphology diminishes with time. With respect to human populations, cross-sectional studies (Hope et al. 2013; Wilson and Manning, 1996) suggest a decrease throughout childhood, an increase at puberty, and then a further decrease in late adolescence/early adulthood. However, FA variance in skeletal traits has been reported to increase with ontogeny in humans and rhesus macaques (Hallgrímsson, 1999), and a longitudinal study of Jamaican youth (Palestis and Trivers 2016) reported a steady increase between 5 and 13 years, after which participants exhibited an elevated rate of FA increase before a slight decline in early adulthood. This pattern was suggested to be reflective of the steady accumulation of developmental errors over time before a rapid increase during puberty as growth rates accelerate. Our study provides longitudinal data relating to an earlier stage of life, which show that FA was lowest at 70 months post-partum, and that it decreased significantly between 9 and 70 months. Although these findings are most consistent with those of Wilson and Manning (1996) and Hope et al. (2013), an explanation that somewhat mirrors the interpretation of Palestis and Trivers (2016) is that FA is initially high due to the extremely rapid growth that is characteristic of early human infancy before it stabilises around $70 \mathrm{M}$ when the rate decelerates prior to onset of puberty (Tanner 1990). This is also reflective of the findings observed by Zelditch et al. (2004) in rodent species, and as such it is possible that similar mechanisms may be responsible, such as a differential in compensating mechanisms between early and late ontogeny. Of course, results pertaining to non-human species must be interpreted carefully when extrapolating to human populations.

An alternative interpretation of these data is that as trait size increases, relative increases in FA may be masked by a general increase in trait size. Without proper allometric controls, this may result in a perceived levelling effect or decrease in FA over time. However, the way in which FA was calculated for the current study should control for noise introduced by trait size. Furthermore, it is difficult to speculate on the impact of a number of external factors that may have influenced FA and its rate of change over time-for example, nutritional factors are known to be strongly associated with human growth patterns (Emmett and Jones 2015; Jasienska, 2013; Lifshitz 2009). Total weight gain after birth has been shown to exhibit a positive correlation with FA in humans (Wells et al. 2006); therefore, variance introduced by dietary changes (e.g., through the development of preference with age or through the influence of school-provided meals) may be implicated in the levelling effect observed at $70 \mathrm{M}$ and is not measured nor controlled for in the current study. However, there is surprisingly little information on how FA changes with time in young infants, so our data provide an interesting preliminary examination, despite these methodological and interpretational issues.

\section{Amniotic Cortisol and FA}

No statistically significant relationship between amniotic cortisol exposure and FA was observed for any specific cohort or when effects were aggregated meta-analytically. Therefore, no empirical evidence is provided in support of foetal cortisol acting as a mediator between maternal stress and offspring FA. However, a lack of evidence is not necessarily evidence of no relationship, and if it is indeed the case that amniotic cortisol exposure relates to FA in offspring there are several potential reasons for why we did not observe the effect here. First, the representativeness of amniotic cortisol measured at a single time point of average cortisol levels throughout gestation is questionable. 
Amniotic cortisol was assayed from samples drawn during amniocentesis, an invasive procedure utilised in cases of increased risk of genetic or chromosomal abnormality. Undergoing amniocentesis can be a stressful experience (Ventura et al. 2012), especially given reports that it is associated with increased foetal mortality (though see Akolekar et al. 2015). It is therefore possible that cortisol levels assayed from samples obtained under these conditions are inflated compared to the average hormonal environment of a given individual, introducing additional noise and limiting the ability to detect a relationship. Evidence in favour of this idea is provided in that maternal circulatory cortisol (itself associated with state anxiety; Sarkar et al. 2006, though see also Baibazarova et al. 2013) for the Portuguese cohort correlated positively with amniotic cortisol. As stress increases maternal cortisol, and increased circulatory cortisol may translate to a concomitant increase in foetal exposure, it appears plausible that the estimation of prenatal cortisol exposure taken under these high stress conditions overestimates the average exposure a foetus experiences during gestation. Moreover, as increased cortisol production is a product of the acute stress response, alongside the amniotic cortisol levels appearing inflated due to the stressful nature of this procedure, they may also not be reflective of true foetal stress as this response may be relatively transient. Acute responses such as these may not be long-lived enough to create meaningful changes in FA.

Although highly statistically significant correlations between maternal and amniotic cortisol were observed, the effects were only moderate in strength: $r=0.429$ and $r=0.420$ for maternal cortisol measured $30 \mathrm{~min}$ before and immediately prior to amniocentesis, respectively. The main regulator of foetal cortisol exposure is thought to be activity of the enzyme $11 \beta$-hydroxysteroid dehydrogenase type 2 (11- $\beta$-HSD 2) (Kajantie et al. 2003). The passage of cortisol through the placenta is partially inhibited by $11-\beta-H S D 2$, which in humans converts excess cortisol into inactive cortisone (Duthie and Reynolds 2013). As 11- $\beta$-HSD 2 levels and functionality are variable between individuals (Kajantie et al. 2003), interventive steps such as this add variance into models of maternal stress, amniotic cortisol and offspring FA, and are not accounted for in our analyses.

Another important consideration is that the calculation of cFA was based solely on digit lengths, and, in some cases only 1 (Portuguese cohort) or 2 (UK cohort) pairs of digits. This is problematic for several reasons. First, digit lengths are not developmentally independent of one another. This means that if some factor affects the developmental trajectory for one digit, it is likely to also influence that of the other digits. This creates a theoretical bias that may be responsible for any effects observed, or indeed, for masking any effects that could have been observed. For this reason, whenever possible, cFA should be calculated from multiple developmentally distinct traits that are still similarly susceptible to the influence of stressors (Palmer and Strobeck 2003). However, amniotic fluid studies are exceedingly rare, and the finger length data presented here were originally collected for a different purpose. We are therefore limited to conducting an opportunistic secondary analysis to test a novel hypothesis.

Second, although the use of only two paired digits (and in the case of the Portuguese cohort for which 2D measures showed signs of directional asymmetry, only one paired digit) may be enough to calculate an FA score, it would certainly be more reliable to include additional traits within the composite (Leung et al. 2000; Palmer and Strobeck 1992, 2003; Van Dongen and Gangestad 2011). The use of one (Portuguese cohort) or two traits (UK cohort) must yield limited information on underlying DI as the differences between right and left for the traits measured can only estimate variance in DI with one or two degrees of freedom (Palmer and Strobeck 2003). This can also be extrapolated to include non-developmentally distinct traits, as by definition these do not truly represent additional information regarding the variance of underlying DI. Thus, it is plausible that the lack of evidence obtained in support of the hypothesis that foetal cortisol exposure and FA are positively correlated can be explained by the imperfect cFA measure utilised in the current study. Alternatively, it is worth noting that many published studies have used only 2D and 4D differentials between left and right hands to calculate composite FA (e.g. Jasienska et al. 2006; Osu et al. 2020; Rogers et al. 2017; Voracek 2009), providing some empirical justification for our use of this method.

Another interpretation of the lack of statistically significant correlations between amniotic cortisol and FA observed here is simply that there is in fact no relationship. For instance, amniotic cortisol may not influence DI and thus would not influence FA; equally, it could be that amniotic cortisol does influence DI, but, as FA provides only a weak proxy for DI, the relationship is not strong enough to be detected with the current level of statistical power. When considering the second explanation, it has been suggested that whilst DI may play a role in the phenotypic variation in expression of FA, it is not the only relevant factor (Palmer and Strobeck 1992). Moreover, increases in FA cannot be parsed between DI and other potential covariates (for example, random wear and tear or random environmental effects) (Palmer and Strobeck 2003). Thus, Palmer and Strobeck (2003) argue that if variance in FA due to DI cannot be distinguished from variance in FA due to other factors, then FA cannot be used as a proper index for DI. That is not to say there is no relationship between FA and DI, just that suggesting FA is produced solely by DI (and thus is a direct proxy for DI) is incorrect. In a recent meta-analysis relating to insect species, Beasley et al. (2013) found that although 
environmental stressors accounted for $36 \%$ of variance in FA, effect sizes were larger in lab studies than field studies, and larger in studies that reported negative correlations with fitness indicators. Such observations appear to support Palmer and Strobeck's (2003) argument that DI accounts for a large proportion (but not all) of the variance in FA, and when more stringent control of extraneous variables is possible, larger effects of FA can be observed. Hence the lack of statistically significant findings here may be due to a lack of proper control of extraneous factors that also influence FA, as well as the relatively weak correlation between FA and DI. With respect to the broader evolutionary significance of these findings, if there is indeed no relationship between amniotic cortisol exposure and FA in the wider population, it is plausible, albeit perhaps unlikely, that acute foetal stress does not cause a related increase in subsequent FA in offspring, and would therefore not translate into meaningful variation in the phenotypic asymmetry of future generations.

\section{Conclusions}

To the best of our knowledge, these data present the first in-depth analysis of prenatal stress hormone exposure and FA in humans. No corroboratory evidence was found for the hypothesised relationship between amniotic cortisol and FA in any of the three cohorts studied, and the meta-analytic effect size estimate was very small and not statistically significant $(r<0.001, p=0.999)$. These findings therefore contrast with the existing literature suggesting that amniotic corticosterone concentrations correlate positively with FA in non-human animals (Eriksen et al. 2003; Newell-Morris et al. 1989; Satterlee et al. 2008). However, the current study did observe positive correlations between the cortisol present in amniotic fluid and the maternal circulation, a finding that replicates those of previous research (Baibazarova et al. 2013; Glover et al. 2009). As maternal blood and salivary samples can be obtained ethically, and with considerably less stress than amniotic fluid, we suggest future studies can provide a stronger test of the hypothesis that prenatal stress hormones influence DI by assaying maternal cortisol during the first and second trimesters of pregnancy and correlating the observed values with a more adequate measure of cFA in their offspring.

\section{Compliance with Ethical Standards}

Conflict of interest The authors declare no conflict of interest.

Open Access This article is licensed under a Creative Commons Attribution 4.0 International License, which permits use, sharing, adaptation, distribution and reproduction in any medium or format, as long as you give appropriate credit to the original author(s) and the source, provide a link to the Creative Commons licence, and indicate if changes were made. The images or other third party material in this article are included in the article's Creative Commons licence, unless indicated otherwise in a credit line to the material. If material is not included in the article's Creative Commons licence and your intended use is not permitted by statutory regulation or exceeds the permitted use, you will need to obtain permission directly from the copyright holder. To view a copy of this licence, visit http://creativecommons.org/licenses/by/4.0/.

\section{References}

Akolekar, R., Beta, J., Picciarelli, G., Ogilvie, C., \& D’Antonio, F. (2015). Procedure-related risk of miscarriage following amniocentesis and chorionic villus sampling: A systematic review and meta-analysis. Ultrasound in Obstetrics and Gynecology, 45(1), 16-26. https://doi.org/10.1002/uog.14636.

Baibazarova, E., van de Beek, C., Cohen-Kettenis, P. T., Buitelaar, J., Shelton, K. H., \& van Goozen, S. H. M. (2013). Influence of prenatal maternal stress, maternal plasma cortisol andcortisol in the amniotic fluid on birth outcomes and child temperament at 3 months.Psychoneuroendocrinology, 38(6), 907-915. https://doi. org/10.1016/j.psyneuen.2012.09.015.

Beasley, D. A. E., Bonisoli-Alquati, A., \& Mousseau, T. A. (2013). The use of fluctuating asymmetry as a measure of environmentally induced developmental instability: A meta-analysis. Ecological Indicators, 30, 218-226. https://doi.org/10.1016/j.ecoli nd.2013.02.024

Benderlioglu, Z. (2010). Fluctuating asymmetry and steroid hormones: A review. Symmetry, 2(2), 541-553. https://doi.org/10.3390/ sym2020541.

Borenstein, M., Hedges, L. V., Higgins, J. P. T., \& Rothstein, H. R. (2009). Introduction to meta-analysis. New York: Wiley.

Boutrus, M., Gilani, S. Z., Alvares, G. A., Maybery, M. T., Tan, D. W., Mian, A., et al. (2019). Increased facial asymmetry in autism spectrum conditions is associated with symptom presentation. Autism Research, 12(12), 1774-1783. https://doi. org/10.1002/aur.2161.

Cohen, J. (1988). Statistical power analysis for the behavioral sciences (2nd ed.). Hillsdale: Lawrence Erlbaum Associates.

de Bruin, E. I., Graham, J. H., Louwerse, A., \& Huizink, A. C. (2014). Mild dermatoglyphic deviations in adolescents with autism spectrum disorders and average intellectual abilities as compared to typically developing boys. Autism Research and Treatment. https ://doi.org/10.1155/2014/968134.

DeBruine, L. (2006). AutoMetric software for measurement of 2D:4D ratios.

Duthie, L., \& Reynolds, R. M. (2013). Changes in the maternal hypothalamic-pituitary-adrenal axis in pregnancy and postpartum: Influences on maternal and fetal outcomes. Neuroendocrinology, 98(2), 106-115. https://doi.org/10.1159/000354702.

Emmett, P. M., \& Jones, L. R. (2015). Diet, growth, and obesity development throughout childhood in the Avon Longitudinal Study of Parents and Children. Nutrition Reviews, 73(S3), 175-206. https ://doi.org/10.1093/nutrit/nuv054.

Eriksen, M. S., Haug, A., Torjesen, P. A., \& Bakken, M. (2003). Prenatal exposure to corticosterone impairs embryonic development and increases fluctuating asymmetry in chickens (Gallus gallus domesticus). British Poultry Science, 44(5), 690-697. https://doi. org/10.1080/00071660310001643660.

Glover, V., Bergman, K., Sarkar, P., \& O’Connor, T. G. (2009). Association between maternal and amniotic fluid cortisol is moderated by maternal anxiety. Psychoneuroendocrinology, 34(3), 430-435. https://doi.org/10.1016/j.psyneuen.2008.10.005.

Golembo-Smith, S., Walder, D. J., Daly, M. P., Mittal, V. A., Kline, E., Reeves, G., et al. (2012). The presentation of dermatoglyphic 
abnormalities in schizophrenia: A meta-analytic review. Schizophrenia Research, 142(1-3), 1-11. https://doi.org/10.1016/j.schre s.2012.10.002.

Graham, J. H., Emlen, J. M., Freeman, D. C., Leamy, L. J., \& Kieser, J. A. (1998). Directional asymmetry and the measurement of developmental instability. Biological Journal of the Linnean Society, 64(1), 1-16. https://doi.org/10.1111/j.1095-8312.1998.tb01530.x.

Hallgrímsson, B. (1999). Ontogenetic patterning of skeletal fluctuating asymmetry in rhesus macaques and humans: Evolutionary and developmental implications. International Journal of Primatology, 20(1), 121-151. https://doi.org/10.1023/A:1020540418554.

Hope, D., Bates, T. C., Dykiert, D., Der, G., \& Deary, I. J. (2013). Bodily symmetry increases across human childhood. Early Human Development, 89(8), 531-535. https://doi.org/10.1016/j.earlh umdev.2013.01.003.

Huizink, A. C., Robles de Medina, P. G., Mulder, E. J. H., Visser, G. H. A., \& Buitelaar, J. K. (2003). Stress during pregnancy is associated with developmental outcome in infancy. Journal of Child Psychology and Psychiatry, 44(6), 810-818. https://doi. org/10.1111/1469-7610.00166.

Jasienska, G. (2013). The fragile wisdom: An evolutionary view on women's biology and health. Cambridge: Harvard University Press.

Jasienska, G., Lipson, S. F., Ellison, P. T., Thune, I., \& Ziomkiewicz, A. (2006). Symmetrical women have higher potential fertility. Evolution and Human Behavior, 27(5), 390-400. https://doi. org/10.1016/j.evolhumbehav.2006.01.001.

Kajantie, E., Dunkel, L., Turpeinen, U., Stenman, U. H., Wood, P. J., Nuutila, M., et al. (2003). Placental 11 $\beta$-hydroxysteroid dehydrogenase-2 and fetal cortisol/cortisone shuttle in small preterm infants. Journal of Clinical Endocrinology and Metabolism, 88(1), 493-500. https://doi.org/10.1210/jc.2002-021378.

King, S., Mancini-Marïe, A., Brunet, A., Walker, E., Meaney, M. J., \& Laplante, D. P. (2009). Prenatal maternal stress from a natural disaster predicts dermatoglyphic asymmetry in humans. Development and Psychopathology, 21(2), 343-353. https://doi. org/10.1017/S0954579409000364.

Körner, L. M., Pause, B. M., Meinlschmidt, G., Tegethoff, M., Fröhlich, S., Kozlowski, P., et al. (2019). Prenatal testosterone exposure is associated with delay of gratification and attention problems/ overactive behavior in 3-year-old boys. Psychoneuroendocrinology, 104, 49-54. https://doi.org/10.1016/j.psyneuen.2019.02.014.

Körner, L. M., Schaper, M. L., Pause, B. M., \& Heil, M. (2020). Parentreports of sex-typed play preference in preschool children: Relationships to 2D:4D digit ratio and older siblings' sex. Archives of Sexual Behavior. https://doi.org/10.1007/s10508-020-01662-6.

Leung, B., Forbes, M. R., \& Houle, D. (2000). Fluctuating asymmetry as a bioindicator of stress: Comparing efficacy of analyses involving multiple traits. American Naturalist, 155(1), 101-115. https:// doi.org/10.1086/303298.

Lifshitz, F. (2009). Nutrition and growth. Journal of Clinical Research in Pediatric Endocrinology, 1(4), 157-163. https://doi. org/10.4008/jcrpe.v1i4.39.

Livshits, G., Davidi, L., Kobyliansky, E., Ben-Amitai, D., Levi, Y., \& Merlob, P. (1988). Decreased developmental stability as assessed by fluctuating asymmetry of morphometric traits inpreterm infants. American Journal of Medical Genetics, 29(4), 793-805. https://doi.org/10.1002/ajmg.1320290409.

Liu, L., Ma, Y., Wang, N., Lin, W., Liu, Y., \& Wen, D. (2019). Maternal body mass index and risk of neonatal adverse outcomes in China: A systematic review and meta-analysis. BMC Pregnancy and Childbirth, 19(1), 105. https://doi.org/10.1186/s1288 4-019-2249-z.
Livshits, G., \& Kobyliansky, E. (1991). Fluctuating asymmetry as a possible measure of developmental homeostasis in humans: A review Author. Human Biology, 63(4), 441-466.

Mittal, V. A., Dean, D. J., \& Pelletier, A. (2012). Dermatoglyphic asymmetries and fronto-striatal dysfunction in young adults reporting non-clinical psychosis. Acta Psychiatrica Scandinavica, 126(4), 290-297. https://doi.org/10.1111/j.1600-0447.2012.01869 .x.

Møller, A. P., \& Swaddle, J. P. (1997). Asymmetry, developmental stability, and evolution. Oxford: Oxford University Press.

Myrskylä, M., \& Fenelon, A. (2012). Maternal age and offspring adult health: Evidence from the Health and Retirement Study. Demography, 49(4), 1231-1257. https://doi.org/10.1007/s1352 4-012-0132-x.

Newell-Morris, L. L., Fahrenbruch, C. E., \& Sackett, G. P. (1989). Prenatal psychological stress, dermatoglyphic asymmetry and pregnancy outcome in the pigtailed macaque (Macaca nemestrina). Biology of the Neonate, 56(2), 61-75. https://doi. org/10.1159/000243104.

Oberlander, T. F., Weinberg, J., Papsdorf, M., Grunau, R., Misri, S., \& Devlin, A. M. (2008). Prenatal exposure to maternal depression, neonatal methylation of human glucocorticoid receptor gene (NR3C1) and infant cortisol stress responses. Epigenetics, 3(2), 97-106. https://doi.org/10.4161/epi.3.2.6034.

Osu, T., Lechler-Lombardi, J., Butler, A., Newman, M., Miłkowska, K., Galbarczyk, A., \& Richards, G. (2020). Fluctuating asymmetry of finger lengths, digit ratio (2D:4D), and tattoos: A pre-registered replication and extension of Koziel et al. (2010). Early Human Development.

Palestis, B., \& Trivers, R. (2016). A longitudinal study of changes in fluctuating asymmetry with age in Jamaican youth. Symmetry, 8(11), 123. https://doi.org/10.3390/sym8110123.

Palma-Gudiel, H., Córdova-Palomera, A., Eixarch, E., Deuschle, M., \& Fañanás, L. (2015). Maternal psychosocial stress during pregnancy alters the epigenetic signature of the glucocorticoid receptor gene promoter in their offspring: A meta-analysis. Epigenetics, 10(10), 893-902. https://doi.org/10.1080/15592294.2015.10886 30.

Palmer, A. R., \& Strobeck, C. (1992). Fluctuating asymmetry as a measure of developmental stability: Implications of non-normal distributions and power of statistical tests. Acta Zoologica Fennica, 191, 57-72.

Palmer, A. R., \& Strobeck, C. (2003). Fluctuating asymmetry analyses revisited. In M. Polak (Ed.), Developmental instability (DI): Causes and consequences (pp. 279-319). Oxford: Oxford University Press.

Phelan, A. L., DiBenedetto, M. R., Paul, I. M., Zhu, J., \& Kjerulff, K. H. (2015). Psychosocial stress during first pregnancy predicts infant health outcomes in the first postnatal year. Maternal and Child Health Journal, 19(12), 2587-2597. https://doi. org/10.1007/s10995-015-1777-z.

Reilly, J. L., Murphy, P. T., Byrne, M., Larkin, C., Gill, M., O'Callaghan, E., et al. (2001). Dermatoglyphic fluctuating asymmetry and atypical handedness in schizophrenia. Schizophrenia Research, 50(3), 159-168. https://doi.org/10.1016/S0920 -9964(00)00044-X.

Richards, G., Gomes, M., \& Ventura, T. (2019). Testosterone measured from amniotic fluid and maternal plasma shows no significant association with directional asymmetry in newborn digit ratio (2D:4D). Journal of Developmental Origins of Health and Disease, 10(3), 362-367. https://doi.org/10.1017/S20401744180007 52.

Richards, Gareth, Browne, W., \& Constantinescu, M. (2020). Digit ratio (2D:4D) and amniotic testosterone and estradiol: An 
attempted replication of Lutchmaya et al. (2004). https://doi. org/10.1101/2020.07.10.197269

Rivet, N., Jamey, C., Reix, N., Kintz, P., Heil, M., Erdmann, K., et al. (2018). Quantifying steroid hormones in amniotic fluid by ultraperformance liquid chromatography and tandem mass spectrometry. F1000Research, 7, 1736. https://doi.org/10.12688/F1000 RESEARCH.15848.1.

Rogers, P., Caswell, N., \& Brewer, G. (2017). 2D:4D digit ratio and types of adult paranormal belief: An attempted replication and extension of Voracek (2009) with a UK sample. Personality and Individual Differences, 104, 92-97. https://doi.org/10.1016/j. paid.2016.07.038.

Ronald, A., Pennell, C. E., \& Whitehouse, A. J. O. (2011). Prenatal maternal stress associated with ADHD and autistic traits in early childhood. Frontiers in Psychology, 1, 223. https://doi. org/10.3389/fpsyg.2010.00223.

Russak, O. D. F., Ives, L., Mittal, V. A., \& Dean, D. J. (2016). Fluctuating dermatoglyphic asymmetries in youth at ultrahigh-risk for psychotic disorders. Schizophrenia Research, 170(2-3), 301-303. https://doi.org/10.1016/j.schres.2015.12.013.

Sarkar, P., Bergman, K., Fisk, N. M., \& Glover, V. (2006). Maternal anxiety at amniocentesis and plasma cortisol. Prenatal Diagnosis, 26(6), 505-509. https://doi.org/10.1002/pd.1444.

Sarkar, P., Bergman, K., Fisk, N. M., O’Connor, T. G., \& Glover, V. (2007). Ontogeny of foetal exposure to maternal cortisol using midtrimester amniotic fluid as a biomarker. Clinical Endocrinology, 66(5), 636-640. https://doi.org/10.111 1/j.1365-2265.2007.02785.x.

Satterlee, D. G., Hester, A., LeRay, K., \& Schmidt, J. B. (2008). Influences of maternal corticosterone and selection for contrasting adrenocortical responsiveness in Japanese quail on developmental instability of female progeny. Poultry Science, 87(8), 1504-1509. https://doi.org/10.3382/ps.2007-00519.

Stiles, J., \& Jernigan, T. L. (2010). The basics of brain development. Neuropsychology Review, 20(4), 327-348. https://doi. org/10.1007/s11065-010-9148-4.

Tanner, J. M. (1990). Foetus into man: Physical growth from conception to maturity. Harvard: Harvard University Press.

Trivers, R., Manning, J. T., Thornhill, R., Singh, D., \& McGuire, M. (1999). Jamaican Symmetry Project: Long-term study of fluctuating asymmetry in rural Jamaican children. Human Biology, $71(3), 417-430$.

Tronche, F., Opherk, C., Moriggl, R., Kellendonk, C., Reimann, A., Schwake, L., et al. (2004). Glucocorticoid receptor function in hepatocytes is essential to promote postnatal body growth. Genes \& Development, 18(5), 492-497. https://doi.org/10.1101/ $\operatorname{gad} 284704$.

Van Dongen, S. (2006). Fluctuating asymmetry and developmental instability in evolutionary biology: Past, present and future. Journal of Evolutionary Biology, 19(6), 1727-1743. https://doi.org/1 0.1111/j.1420-9101.2006.01175.x.
Van Dongen, S., \& Gangestad, S. W. (2011). Human fluctuating asymmetry in relation to health and quality: A meta-analysis. Evolution and Human Behavior, 32(6), 380-398. https://doi.org/10.1016/j. evolhumbehav.2011.03.002.

van Os, J., \& Selten, J.-P. (1998). Prenatal exposure to maternal stress and subsequent schizophrenia. British Journal of Psychiatry, 172(4), 324-326. https://doi.org/10.1192/bjp.172.4.324.

van Valen, L. (1962). A study of fluctuating asymmetry. Evolution, 16(2), 125-142. https://doi.org/10.1111/j.1558-5646.1962.tb032 06.x.

Ventura, T., Gomes, M. C., \& Carreira, T. (2012). Cortisol and anxiety response to a relaxing intervention on pregnant women awaiting amniocentesis. Psychoneuroendocrinology, 37(1), 148-156. https ://doi.org/10.1016/j.psyneuen.2011.05.016.

Ventura, T., Gomes, M. C., Pita, A., Neto, M. T., \& Taylor, A. (2013). Digit ratio (2D:4D) in newborns: Influences of prenatal testosterone and maternal environment. Early Human Development, 89(2), 107-112. https://doi.org/10.1016/j.earlhumdev.2012.08.009.

Viechtbauer, W. (2010). Conducting meta-analyses in R with the metafor package. Journal of Statistical Software, 36(3), 1-48. https:// doi.org/10.18637/jss.v036.i03.

Voracek, M. (2009). Who wants to believe? Associations between digit ratio (2D:4D) and paranormal and superstitious beliefs. Personality and Individual Differences, 47(2), 105-109. https://doi. org/10.1016/j.paid.2009.01.051.

Walker, D. A. (2003). Converting Kendall's tau for correlational or meta-analytic analyses. Journal of Modern Applied Statistical Methods, 2(2), 525-530. https://doi.org/10.22237/jmasm/10676 46360.

Weinstock, M. (2005). The potential influence of maternal stress hormones on development and mental health of the offspring. Brain, Behavior, and Immunity, 19(4), 296-308. https://doi. org/10.1016/j.bbi.2004.09.006.

Wells, J. C. K., Hallal, P. C., Manning, J. T., \& Victora, C. G. (2006). A trade-off between early growth rate and fluctuating asymmetry in Brazilian boys. Annals of Human Biology, 33(1), 112-124. https ://doi.org/10.1080/03014460500480391.

Wilson, J. M., \& Manning, J. T. (1996). Fluctuating asymmetry and age in children: Evolutionary implications for the control of developmental stability. Journal of Human Evolution, 30(6), 529-537. https://doi.org/10.1006/jhev.1996.0041.

Yeo, R. A., \& Gangestad, S. W. (1993). Developmental origins of variation in human hand preference. Genetica, 89(1-3), 281-296. https://doi.org/10.1007/bf02424521.

Żądzińska, E., Kozieł, S., Kurek, M., \& Spinek, A. (2013). Mother's trauma during pregnancy affects fluctuating asymmetry in offspring's face. Anthropologischer Anzeiger, 70(4), 427-437. https ://doi.org/10.1127/0003-5548/2013/0383.

Zelditch, M. L., Lundrigan, B. L., \& Garland, T., Jr. (2004). Developmental regulation of skull morphology. I. Ontogenetic dynamics of variance. Evolution \& Development, 6(3), 194-206. 